\title{
BMJ Open Implementing online evidence-based care pathways: A mixed-methods study across primary and secondary care
}

\author{
Joy Akehurst, ${ }^{1}$ Zeibeda Sattar, ${ }^{2}$ Isabel Gordon, ${ }^{1}$ Jonathan Ling ${ }^{1}$
}

To cite: Akehurst J, Sattar Z, Gordon I, et al. Implementing online evidence-based care pathways: A mixed-methods study across primary and secondary care. BMJ Open 2018;8:e022991. doi:10.1136/ bmjopen-2018-022991

- Prepublication history for this paper is available online. To view these files, please visit the journal online (http://dx.doi. org/10.1136/bmjopen-2018022991).

Received 18 March 2018 Revised 6 October 2018 Accepted 2 November 2018

Check for updates

(c) Author(s) (or their employer(s)) 2018. Re-use permitted under CC BY-NC. No commercial re-use. See rights and permissions. Published by BMJ.

${ }^{1}$ University of Sunderland, Sunderland, UK

${ }^{2}$ Northumbria University, Newcastle upon Tyne, UK

Correspondence to Professor Jonathan Ling; jonathan.ling@sunderland.ac.uk

\section{ABSTRACT}

Aim To understand what contextual influences, mechanisms and outcomes affect the implementation and use of localised, online care pathways (HealthPathways) in primary and secondary care.

Design and procedure Mixed-measures design. Quantitative data included number of page views and conditions viewed. Qualitative data from semistructured interviews and focus groups were gathered over a 6-month period, and analysed using NVivo software. Setting The first HealthPathways UK site, South Tyneside, England.

Participants General practitioners, nurses, practice managers, hospital consultants and system leaders (managers, commissioners) ( $n=76)$.

Results Use of the pathways significantly increased over time. Themes were developed showing how online care pathways were used-leadership, pre-existing networks and relationships; development of systems and processes for care pathways, the use of online care pathways to support decision-making and referral, and perceived availability of resources. Inter-related themes were arranged into configurations consisting of contextual influences, mechanisms and outcomes. Recommendations were made for future implementations, such as improved data collection processes to understand how and why there was variance in the use of pathways.

Conclusions This study was early in the implementation process; however, emerging themes will facilitate the future implementation and use of online care pathways. Recommendations are made for further research to include other health and social care users and patients to inform future developments.

\section{INTRODUCTION}

The National Health Service (NHS) Five Year Forward View ${ }^{1}$ emphasises integration and collaboration across a whole system to make best use of resources and improve the patient care experience. Workforce development and technology have been identified as two factors which facilitate integrated working, reduce variability in care and improve efficiency. ${ }^{2-7}$ Online care pathways enable rapid access to evidence-based resources for professionals to support decision-making, treatment, care planning and referral, for

\section{Strengths and limitations of this study}

This is the first UK study of the implementation and use of localised, online care pathways (HealthPathways) across health and social care.

- The study was time-limited, with limited quantitative data available for analysis.

- Data relating to referrals made following use of HealthPathways would have enabled a deeper understanding of variance in use of agreed care pathways. These data could have also been used as part of the interviews and focus groups, to draw out further learning.

- The focus was mainly on general practitioner users and system leaders.

- Future research should focus on a wider range of HealthPathways users including nurses, allied health professionals and patients.

elective and non-elective care. ${ }^{8}$ Clinical pathways can also improve communication, teamwork and care planning in multidisciplinary teams, which in turn helps coordination of care across organisations. ${ }^{9}$ Evidence-based information used at the point of care has also been reported to mitigate risk, be effective in improving patient care outcomes and reduce cognitive overload which can lead to medical errors. ${ }^{10}{ }^{11}$ The concept of 'task transfer' changing roles and responsibilities is also evident within policy and literature on care pathways, for example, from medical to nursing and allied health professionals. ${ }^{12} 13$

Canterbury District Health Board in New Zealand is internationally recognised for transforming its health system through integration and collaboration, reducing acute hospital admissions, treating more patients outside of hospital, with strong staff engagement across the health and social care system. ${ }^{14}{ }^{15}$ Part of this whole system approach is HealthPathways, ${ }^{16}$ an online repository of $>550$ evidence-based care pathways with the aim of providing the right care, in the right place at the right time, to reduce variability and increase efficiency. HealthPathways 
provides a combination of clinical guidelines, local process information (for referrals and diagnostics and associated clinical responsibilities), service directory information and patient information. For example, a primary care clinician can access a pathway for cognitive impairment, which includes which tests/assessments should be conducted in a primary care setting (agreed between primary and secondary care clinicians), differential diagnoses, when and how to refer to a local specialist, what local services there are to support the patient and their family, and information about the condition for patients. HealthPathways has been implemented in 33 sites in New Zealand and Australia, in largely mixed private and publicly funded health and social care economies, covering $>25$ million people. ${ }^{17} 18$

South Tyneside in the northeast of England is an NHS Integrated Care Pioneer ${ }^{19}$ and the first UK site to implement HealthPathways. HealthPathways went live in August 2016 with 50 care pathways accessed via a weblink.

The aim of the study was to understand what contextual influences, mechanisms and outcomes affect the implementation and use of localised, online care pathways (HealthPathways) across primary and secondary care for this national pilot site.

\section{METHODS}

The study methodology draws on the realist approach to evaluation, ${ }^{20}$ providing an explanatory analysis aimed at showing what works for whom, under what circumstances, in what respects and how in order to provide an in-depth understanding of an intervention and how it can be made to work most effectively. In this paper, we focus on examining the relationship between the context in which the HealthPathways system is applied, the mechanisms by which it works and the outcomes which are produced. ${ }^{21}$ This approach is useful as a pragmatic way of understanding the contextual complexity within which interventions can take place and influences over how they work, such as with HealthPathways where there may be multiple external variables or where there is an evolving intervention. $^{2022} 23$
Data gathered included HealthPathways usage data, interview and focus group data. Semistructured topic guides developed for one-to-one interviews and focus groups included broad issues identified through documentary analysis and in current evidence on clinical pathway systems and HealthPathways. Focus group and interview data were collected to provide rich data across disciplines and team members.

Sets of themes generated via analysis using NVivo software were arranged as configurations under the headings of context, mechanisms and outcomes (see table 1). During manual analysis, themes found to be inter-related both within these headings and across each configuration were included.

\section{Sampling and recruitment}

Ten general practitioner (GP) practices in South Tyneside were purposively sampled and invited to participate in interviews and focus groups. Participants were selected based on variables such as geographic location, size of practice, years of experience as GPs and whether they were currently users of HealthPathways, to ensure a wide range of views. Four of the practices were unable to participate within the time frame of the study due to external pressures (planned Care Quality Commission inspection, practice mergers). Hospital consultants were also invited to participate. Participants $(n=76)$ included GPs, district nurses, practice nurses, practice managers, healthcare assistants and system leaders. System leaders (clinical and non-clinical) either had roles in the strategic or operational development of HealthPathways, in individual pathways as subject matter experts or were clinical editors (two GPs who worked part-time to support the writing of pathways). Three of the system leaders had clinical and management responsibilities (hospital consultants). One system leader was from a social care background. Four focus groups of GP teams were held with a total of 45 participants.

\section{Data collection}

Quantitative

Data were generated from the HealthPathways system using Google Analytics. Data included number of

\begin{tabular}{|c|c|c|c|}
\hline Configuration & Context & Mechanism & Outcome \\
\hline 1 & $\begin{array}{l}\text { Strategy } \\
\text { Ethos }\end{array}$ & Leadership & Uptake \\
\hline 2 & $\begin{array}{l}\text { Pre-existing strong networks and } \\
\text { relationships }\end{array}$ & $\begin{array}{l}\text { Canterbury District Health } \\
\text { Board input }\end{array}$ & First impressions from introduction phase \\
\hline 4 & Decision-making and referrals & $\begin{array}{l}\text { HealthPathways for referrals } \\
\text { Two-way communication } \\
\text { Task transfer }\end{array}$ & $\begin{array}{l}\text { Compliance with pathways } \\
\text { Specific pathway outcomes - patient } \\
\text { stories }\end{array}$ \\
\hline
\end{tabular}




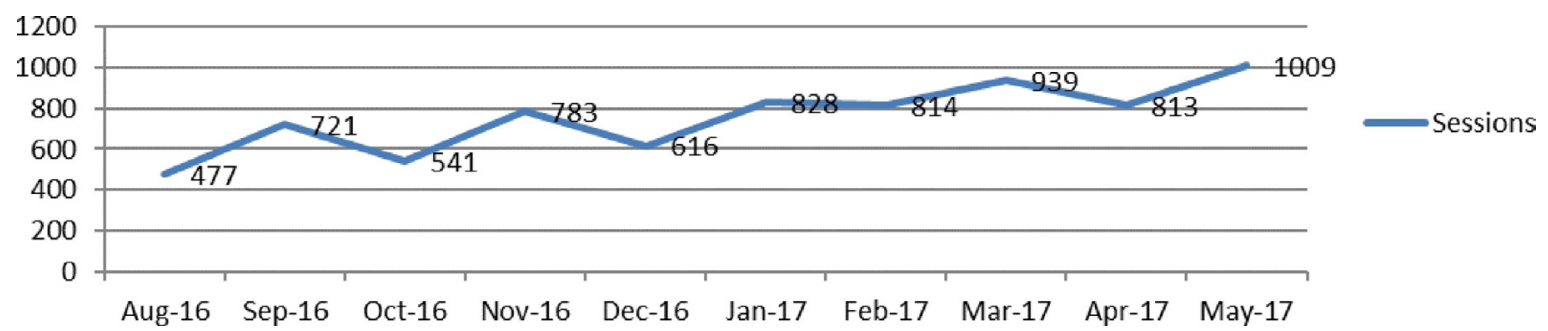

Figure 1 Number of sessions on South Tyneside HealthPathways from 16 August 2016 to 31 May 2017. A session is defined as a login to the online care pathway repository.

HealthPathways sessions and page views, number of users and use of specific pathways. Data were collected between 19 August 2016 and May 2017.

\section{Qualitative}

Data from 15 semistructured interviews with system leaders and GPs as well as 4 focus groups $(n=45)$ of GP practice team members were conducted. The interviews and focus groups took place in general practice meeting rooms, or sites most suitable for participants and lasted between 30 and $90 \mathrm{~min}$. Qualitative data were digitally recorded and transcribed verbatim prior to analysis by the research team. Notes from observations of two project meetings $(n=8)$ between system leaders, commissioners and the research team were taken to support analysis.

\section{Data analysis}

\section{Quantitative}

Data on use of HealthPathways were collated as a means of understanding the level of engagement. Number of sessions, page views and users were all collected monthly and analysed by correlation.

\section{Qualitative}

Thematic analysis was carried out using the qualitative research software package Nvivo; manual analysis was used to refine the initial findings. Context, mechanism and outcome configurations were developed from the themes emerging from the data. To improve validity, coding and interpretations were discussed by members of the research team (JA, ZS, IG) at regular meetings during analysis.

\section{Report writing}

We used the SQUIRE checklist when writing our report. ${ }^{24}$

\section{Patient and public involvement}

Patients were not involved in developing this initial study as the focus was on clinician use of online health and social care pathways.

\section{RESULTS}

\section{Quantitative data}

There were 130 GPs in South Tyneside who were given access to HealthPathways via a weblink for each practice. Other users in the practice also potentially had access.

In total, there were 7544 sessions on HealthPathways and 37186 page views in the study period. The number of sessions increased over this period from 477 to 1009, and the number of page views from 3309 to 4766 (figures 1 and 2). There was a steady increase in use, suggesting the pathways were being accessed more regularly by practitioners. Over time, there were significant increases in numbers of both sessions $(\mathrm{r}=0.843, \mathrm{n}=10, \mathrm{p}=0.02)$ and users $(r=0.794, n=10, p=0.006)$, with a trend towards there being an increase in page views $(r=0.627, n=10, p=0.052)$.

In May 2017, 252 users had logged onto HealthPathways (figure 3). As there are 130 GPs in South Tyneside, there were potential multiple logins or other users than GPs. At the time of writing, the ability to determine who these were was unavailable.

\section{Qualitative data}

Themes that illustrate how HealthPathways might be implemented and used over the long term were identified. These themes are summarised as configurations in table 1 within the framework of context, mechanisms and outcomes set out in the realistic evaluation model. ${ }^{22}$

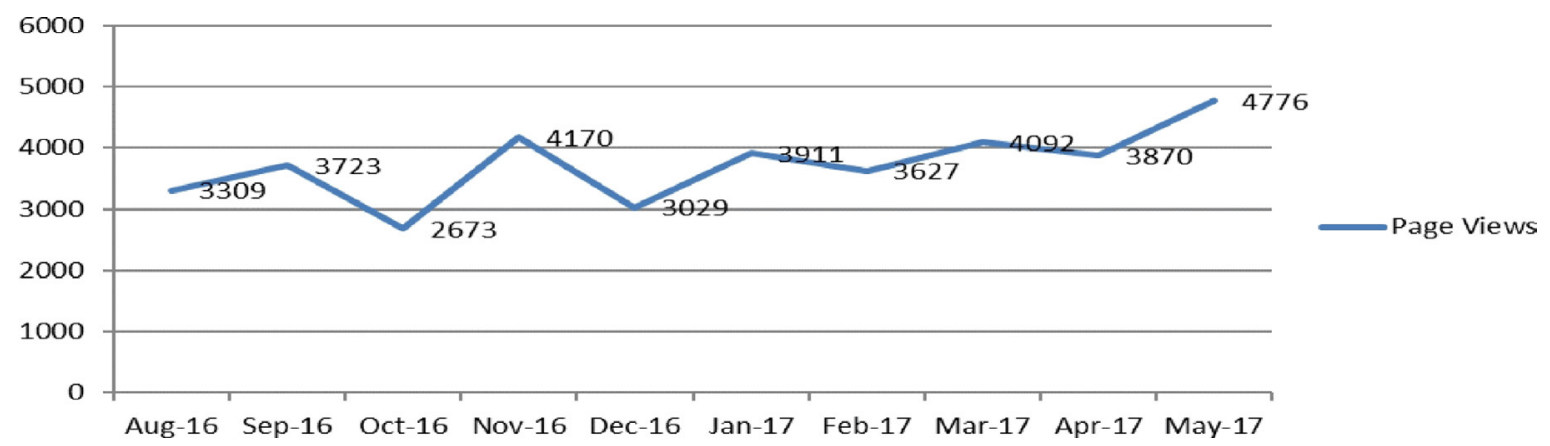

Figure 2 Number of page views of South Tyneside HealthPathways from 16 August 2016 to 31 May 2017. 




Figure 3 Number of users from August 2016 to May 2017 for South Tyneside HealthPathways.

Themes reported under 'Context' refer to the organisational structure, systems and ethos within which the HealthPathways system was embedded. Themes under the heading 'Mechanism' refer to processes by which the HealthPathways system was used and developed by participants. 'Outcome' themes refer to how participants perceived the HealthPathways system to have influenced particular contexts.

The configurations derived from thematic analysis of the data are described below.

\section{Configuration 1: strategy-leadership-uptake}

This configuration focuses on the introduction phase and brings together the contextual theme of strategy, the mechanism theme of leadership and the outcome theme of uptake. These closely interlinked themes encompass how HealthPathways was set up within the localised user community.

The strategy for HealthPathways focused on what implementing it meant for the health and social care system and what motivated people to engage with it. Many system leaders were aware that HealthPathways was part of a system-wide transformation programme and were optimistic that it would help reduce pressure due to lack of resources in the locality. One strategy for implementation was a launch event which was well-attended $(n=180)$ that aimed to engage clinical users with the idea of HealthPathways and encourage them to use it.

I think the launch that we did ... I think, really did go a long way to selling it to people. (System leader, I1, p. 5)

A fundamental part of the HealthPathways strategy was its underpinning ethos. The way this was presented to users was a key leadership tool both at the launch and in ongoing project meetings and clinical forums. The ethos was focused on working together towards a common goal with an absolute commitment to partnership across all professional groups involved in the development, use and maintenance of the HealthPathways system.

We're talking about a way of working, we're talking about empowering clinicians and saying just do what's right—-they do that. (I2, p. 5)

The optimism expressed by system leaders that the ethos of HealthPathways would be integrated into the working system did not always come across from those using the system, who were faced with practical challenges such as time pressures and using the system while multitasking. The challenges of getting people on board with this change in thinking were also acknowledged by the Canterbury team leader:

It's getting your people engaged in the change that will make a difference over time. That's the experience of Canterbury. It comes back to that cultural underpinning stuff again. (Canterbury team leader, I3, p. 13)

An early introduction to this ethos as an integral part of the HealthPathways working culture was seen by system leaders to reinforce users' faith that HealthPathways would work. Overall, system leaders including GPs and hospital consultants perceived themselves to be singing from the same hymn sheet' (system leader, I6, p. 12) in advocating the philosophy, values and open work ethic of HealthPathways.

So there was a sense of real commonality, even though, very clearly, they're halfway round the world, but there was an obvious synergy in what they were doing and had done over the previous ten years and what we were trying to do in the borough. (System leader, I4, p. 2)

Leadership and exposure to leaders appears to have had a positive and motivational effect on potential users. Leaders included the Canterbury Team, system leaders, clinical editors and subject matter experts in primary and secondary care. Several participants commented on the importance of having a local champion. System leaders also recognised that gaining clinicians' interest and understanding of HealthPathways and the ethos underlying it could be a challenge, but integral to its successful implementation.

... persuasion of the secondary care community that they should give their time to come and be involved in these [launch/training events], is quite a significant ask ... they totally need to get this. That is a challenge. (System leader, I4, p. 14)

Some comments suggested a need for careful management of users' expectations about HealthPathways during the set-up phase such as how it could be used, time commitment and how HealthPathways would benefit users. 
R2: The CCG had a big roll-out event that we were all invited to come to.

R1: I think it was a bit over-sold. Because it [HealthPathways] doesn't give us all the answers that we want ... (GP, I15, p. 4)

The clinical editors and subject matter experts felt they were developing a key leadership role in service development which they had not anticipated and were anxious about:

We've been asked to look at when we're writing a pathway, ... are there areas where we can see that we could try and make a change? I think sometimes that's felt a little bit daunting. (Clinical editor, 19, p. 4)

The uptake of HealthPathways appeared to be influenced by their initial perceptions of the system gained from the introduction phase. They commented on the launch event, training provided, education forums and first experiences of using the system and seemed to value the training, strong leadership and fast responses when providing feedback. They recognised that their views were formed on the basis of HealthPathways being newly introduced and that gaining trust in the system may take time.

I fired off an email and within 24 hours the editors had looked at it, dealt with it, said thank you, and it was updated and it was working beautifully. So they are incredibly responsive if people are willing to [try]. (GP, I7, p. 7)

Participants who did not attend the launch or who had not met those who had successfully implemented HealthPathways appeared less enthusiastic about system transformation opportunities and benefits for patients. For some clinicians it was gaining insight from peers which was most persuasive. For example, practices where GPs were developing pathways their GP colleagues seemed to be more engaged with HealthPathways:

We've had quite a lot of information leading up to Health Pathways, when we've been to talk to some of the CCG. [...] One of the GPs in the practice works at the CCG as well. So, we've had quite a lot of information ... (GP, 14, p. 1)

Practical suggestions were made to both improve and ensure the uptake of HealthPathways i, for example, linking HealthPathways to the patient health record or the referral system, both of which would save users time. System leaders acknowledged clinicians' lack of time in their strategy to 'only push where the open door is' (I2b, p. 6). Time delays in developing and agreeing pathways with hospital medical staff were reported by GPs involved as subject matter experts as having the potential to affect uptake.
Configuration 2: pre-existing strong networks and relationships- Canterbury District Health Board input-first impressions

This configuration describes how the presence of existing working relationships (context) set the scene for the involvement of the Canterbury team in launching HealthPathways and training people to lead and use the system (mechanisms). Clinicians' first impressions of HealthPathways from the introductory phase were outcomes.

Participants across groups described the pilot area as having particularly strong networks that were in place before HealthPathways was introduced. These influenced overall impressions of HealthPathways from the outset. They recognised this context as unusual and felt this was a key factor in the effective implementation of HealthPathways locally. The Canterbury team were struck by the level of formal and informal dialogue between stakeholders:

.... they'd already worked very hard to develop a lot of the relationships between the key players, which was the prime reason that we came here. And they had some leaders in place who were motivated to [promote] change. (Canterbury system leader I3)

There was also optimism about the role of educational forums where relationships had already developed between clinicians; these were felt to be potentially useful for giving feedback on the development, use and outcomes of pathways.

I became much more enthusiastic about Health Pathways after going to a care of the elderly meeting ... that certainly contributed to developing a pathway .... which is possibly more important than the actual guideline. (GP, I7, p. 2)

Involvement of the Canterbury team was seen as fundamental in helping establish use of the HealthPathways system and reinforcing what worked for them in a new locality. For example, system leaders in South Tyneside commented on how the Canterbury Team helped realise their existing networks and relationships:

The major thing that, I think, we took from Canterbury, was really about the partnership element. So, this was not about organisational barriers or boundaries, it was really about trying to open up much more and be part of the system and work a system together ... (System leader, I4)

Participants' first impressions of HealthPathways revealed differences in opinion between and within groups about to start using the system. Those from both clinical and non-clinical settings reported system fatigue and practitioners' lack of time being the context for its introduction.

I'm not asking it to be discussed every month [at practice meetings] just a little reminder to keep letting people know it is there. Otherwise it will just dwindle away like everything else. (GP, I10, p. 5) 
Clinicians' reasons to use HealthPathways varied, for example, some hospital consultants saw the trade-off between investing time and the resulting information available as beneficial, some GPs saw it as an information resource and others expressed caution about promoting the use of HealthPathways to reduce secondary care demand.

It's an accessible way of me getting the answers that I want that are going to help me with a particular patient I have sat in front of me. And that's not always about reducing demand on secondary care ... (GP, I12, p. 2)

However, others were unsure of the impact of HealthPathways on their practice at this early stage:

I don't think it's made a massive difference to my referral patterns. But that might be if I used it a bit more, it could. I don't know- [Name] is a bit more of an enthusiast. But it's only just started. (GP, I7, p. 4)

In contrast, both clinical and non-clinical system leaders seemed more confident in the usage of HealthPathways and felt HealthPathways was a way to improve efficiency by preventing illnesses progressing by speeding up referrals to the correct place, thereby saving money rather than cutting back on services.

... you can see that they [GPs] are using it. They appreciate that the information is in one central repository and they are referring when needing to refer and doing what actually should be done in primary care. (System leader/hospital consultant, I8, p. 2)

There were some tensions between GPs and hospital consultants in their perceptions of how HealthPathways was used and the benefits it offered them which may have an impact on long term use. There was concern about creating a them and us culture' (GP, I17, p. 14) with the patient in the middle, when the intended seamlessness between primary and secondary care did not work. Another concern was whether input into the development of a pathway was required from both primary and secondary care, or from clinicians with different clinical backgrounds:

The pathway sits, sort of, between myself and with [Name], who's one of our elderly care consultants. So, it had to be done jointly, and again there were complications in terms of input from my side, from her side, and then pulling all that together and where the pathway sits neatly in speciality, perhaps, it's a bit more straightforward. (System leader, 19)

Some secondary care participants expressed concerns that the online care pathways would mean a potential shift of resources to primary care from the hospital, despite the hospital having a contract, which was not at that time funded by activity levels. Some GPs wanted to use the care pathways repository as a tool to demonstrate their continued professional development, as some clinical guidelines systems can; it was thought this may encourage more use and promote GPs' engagement with HealthPathways:

The reason that I know other doctors like [another online system] is because it logs that you've been in. So, at any stage you can say 'Give me a history of the things that I've looked up.' And it will give you [that] ... So that is useful for recording of your continual professional development ... (GP, 12)

Other GPs saw HealthPathways as a directory of services or clinical resource rather than a local team approach to developing referral pathways. There were also GPs who saw it as a tool to support effective and efficient decision-making, to make better use of resources in the health and social care system, and to improve patient care. When clinicians were asked if HealthPathways had improved their relationships with patients, only a small number of users had engaged in any discussion about care pathways with a patient; however, some saw the potential:

If we genuinely believe that shared decision-making and people being involved in their own care, enables and empowers them and increases satisfaction, compliance, then me being able to go through a pathway with someone, I think would be helpful ... (GP, I7)

While clinicians' views were mixed there was a common element of uncertainty that HealthPathways could be a sustainable new approach to delivering care at this stage of its development.

Configuration 3: systems for development of HealthPathways and individual pathways_-development process, training, practical use, pathways-feedback

This configuration focusses on the system factors such as the way pathways were set out, developed and used by participants. The context themes are systems for development of HealthPathways and individual pathways, the mechanism themes are the development processes and practical usage of the pathways and the outputs are feedback about pathways.

The systems for development consisted of HealthPathways and individual pathways. Fifty pathways were prioritised for initial development based on existing Canterbury pathways which could be localised, or which were 'quick wins' for the system as they did not need localisation, and which HealthPathways users identified would be most useful from the onset (table 2). Getting the first 50 pathways ready for the launch proved challenging for clinical editors due to poor planning, lack of information about how to do it and a lack of time and staff.

I knew I shouldn't have been up at two in the morning doing it ... we were just told to get out and go and do it ... Because we weren't told, right, these are the steps, this is the very detailed process ... And it just caused delay and challenge. (System leader, I2, pp. 13-14) 
Table 2 The 10 most frequently visited pathways in South Tyneside (localised and non-localised), May 2017

\begin{tabular}{llll}
\hline Localised pathways & Page views & Non-localised pathways & Page views \\
\hline Chronic obstructive pulmonary disease & 428 & End of life & 105 \\
Heart failure & 406 & Hyperlipidaemia & 74 \\
\hline 2-week wait suspected cancer referrals & 350 & $\begin{array}{l}\text { Differentiating asthma from chronic obstructive } \\
\text { pulmonary disease }\end{array}$ & 58 \\
Atrial fibrillation & & Insulin-starting and ongoing management \\
Integrated care teams & 321 & Type 2 diabetes - newly diagnosed & 56 \\
Deep vein thrombosis & 306 & Abnormal liver function tests & 53 \\
Non-acute cardiology & 250 & Advanced care planning & 52 \\
Chronic kidney disease in adults & 214 & Cardiovascular risk assessment & 52 \\
Diabetes continuing care & 213 & Spirometry interpretation & 49 \\
Diabetes diagnosis & 201 & Urinary tract infection in adults & 45 \\
\hline
\end{tabular}

There were different approaches to the development process for pathways. GPs had mixed views on the process of inputting information and getting feedback, some found it easy and underlined the importance of a partnership approach to developing the system locally; others found the differences in medical terminology or time delay of working with editors in New Zealand frustrating.

The problem is, currently they're not all localised to [the local area]. And that can be very frustrating, to go in and find that you've got this problem and the way to manage it is to refer it to Canterbury Health Board. (GP, I7, p. 3)

Some subject matter experts (consultants and GPs) discussed the proposed content via email rather than planned subject matter group meetings; however, this approach was seen as being less productive and taking longer than face-to-face discussion. Using existing clinical education meetings was suggested as a better place to discuss and agree care pathways, and was felt would also improve integrated working across the system.

It was important to users that their views were listened to during the development of pathways. HealthPathways has a user feedback system called DOT which enables users to $\log$ queries or suggested amendments. Users reported that they were more likely to engage with HealthPathways if they received prompt and positive feedback, when they logged a query, as this reinforced their clinical expertise seeing their own impact on the system was also a factor that motivated them.

... with the blood pressure targets for diabetes .... they changed them within 24 hours, saying thanks for identifying it, and letting us know what the real [correct] ones are. We've changed it. And it does make you buy in, that sort of thing. (GP, I7, p. 7)

Challenges during the development stage of HealthPathways included a lack of clinical time to develop, agree and use HealthPathways. This was particularly challenging for clinical editors who reported that they needed more time than planned to set up the initial pathways required for the launch. This was because their role increasingly had a service development function to ensure services were configured in line with the evidence base in HealthPathways. This affected timely delivery of new pathways and meant additional costs for others in the system.

The bottom line is things that would facilitate the use of Health Pathways, would be having all the health pathways localised at the time of going live. I appreciate there's a financial constraint to doing that ... (GP, I7, p. 3)

At this early stage, it was unclear how these costs might be captured or analysed to be able to demonstrate future return on investment. Other clinicians also felt they did not receive additional time or financial resources to contribute to the development of pathways:

In terms of practical usage, clinicians' motivation seemed to be influenced by the way pathways were set up and how they could be accessed.

One of the things that I'm not keen on with the pathways, is there are ... There are quite a lot of ifs, buts and therefores. So you can't follow one through with the patient because it is designed for a medic. And you can't pick one up that you've not used before mid-consultation, because there isn't a natural flow to them. There's almost too much information. (GP focus group, I7, p. 5)

Training in the use of HealthPathways was made available for all GP practices, but many felt the system was intuitive and easy to use without training. Some of these GPs reported using HealthPathways as a resource directory rather than being involved with the development of pathways. Non-clinical system leaders who were not used to NHS terminology found this aspect of using HealthPathways took time to learn. 
If you're from that kind of industry and you already know the terminology and things like that, that's half the battle. (System leader, I1, p. 4)

The use of localised pathways was greater and related to the most common conditions encountered. Some GPs reported however that if a condition was commonly occurring, they would be less likely to view the pathway, as they felt they knew what the correct pathway was. There were mixed views about using the non-localised, New Zealand, pathways. Some felt this was a 'trust' issue, that is, whether the clinical evidence base would be the same in the UK. The fact that some pathways were not localised, accurate (in terms of UK practice) or complete was perceived as a potential barrier to using the system to its full capacity:

I mean, of course there are lots which are not relevant to us, because it's the New Zealand stuff. Which, again, are referred to for the clinical information, but not for the referrals and stuff like that ... (GP, I10, p. 4)

Others however reported the clinical content of pathways which were already developed was as good as, if not better than, others developed elsewhere in the UK.

In terms of practical use, HealthPathways was not currently integrated into existing patient health and social care record systems. This meant users needed to access HealthPathways via an additional screen. This switching between screens reduced use of HealthPathways. Using HealthPathways was also felt to be more difficult if a patient was present or when consulting with patients with multiple health conditions.

It was a patient who had a number of different health conditions. So, one of their health problems meant that the straightforward Health Pathways pathway would have been inappropriate in their case ... (GP, I14, p. 5)

At the time of the study, the design of the HealthPathways system was such that data about individual use were not available to system leaders; these would have been useful to understand patterns of use, and to identify areas for further development both in terms of pathways and training for users.

Configuration 4: decision-making and referrals-two-way communication, task transfer, compliance-specific pathway outcomes

This configuration focuses on the use of HealthPathways in clinical practice, where context themes were decision-making and referrals, mechanisms were task transfer and two-way communication and outcomes were compliance with pathways or specific outcomes.

A key objective of HealthPathways is to support decision-making and referral to enable more care to be delivered in primary care, community care and patients' homes, and to make best use of resources across the system. At the time of data collection, the HealthPathways system was still relatively new, and users reported they still conformed to their standard decision-making about referral. However, users reported they used HealthPathways to check whether the pathway had changed or to confirm their knowledge (eg, if they were newly qualified or new to the area). Secondary care clinicians, however, reported there was not always compliance with pathways as they were still receiving referrals despite the pathway stipulating referral was inappropriate:

I get the feeling that not all of them are using it. I think there are certain conditions that I deal with, where if you follow the pathways it's all there. But we're still getting referrals for some of these cases where ..., if you follow the pathways, you wouldn't refer ... (System leader, 19)

In addition, the challenges of complying with newly agreed pathways were also articulated by a secondary care clinician:

I was discharging a patient-very stable, on insulin, kidney disease stable-who'd I'd looked after for 12 years. And I made it clear, you know, 'discharging you back to your GP, but you've got a safety net' etc. ... I think that [HealthPathways] really is the thing which has helped me to do that. And it can be difficult .... because you do build your relationship with patients. But ultimately, if your clinics are overbooked, are you really doing a service? (System leader, I8)

Built into HealthPathways care pathways is the concept of 'task transfer' from hospital consultants to GPs as part of the vision for more care outside of hospital. An emerging theme was of task transfer from GPs to nurse practitioners and district nurses. The small number of nurses in focus groups who had used HealthPathways felt they would engage more if HealthPathways could provide more clarity about care pathways for patients who were discharged from hospital care, as well as for referral in:

In principle, the system is a one-way system. So, it's [HealthPathways] designed to help secondary care by us following pathways that they set out for us to follow to send the patient in. Perhaps one of the improvements would be if primary care wrote the pathway for secondary care to send people back out. (Nurse practitioner, 17)

The importance of two-way communication, task transfer and the role of HealthPathways to prevent a 'them and us' culture was a common theme:

I appreciate the volume of work that they [hospital consultants] do, but I don't know exactly everything that they do. ... if this is truly about integrated care-if this is truly about making things better for everyone within the NHS, then actually it's not working. Because if it's creating a them-and-us culture ... That's no interface at all ... (GP, 17) 
Quantitative data on specific pathway outcomes were unavailable as the system was not yet sufficiently developed to be able to do this; however, there were several comments from HealthPathways users about when a pathway had supported the right care for a patient and suggesting there could be positive outcomes for the patient.

I'd not seen [the patient] for a very long time ... and gave them up to date information about that pathway and what to prescribe so it was useful. (GP, I14, p. 3)

\section{DISCUSSION}

This study is the first to examine the implementation of HealthPathways in the UK and was conducted at the national pilot site. The study started when HealthPathways had only been operational for 4 months and yet we found there was an increasing number of HealthPathways users and a number of useful themes from the qualitative data which will support other implementations of online care pathways. HealthPathways had been in place for 11 months when the study concluded, so only initial recommendations on improving uptake were suggested. Implementing change, particularly at scale, often require time and targeted efforts, a finding which has been reflected in other studies. ${ }^{625}$

The four configurations were developed in line with a realist approach to explore practical ways of supporting future implementations of online care pathways across health and social care. These configurations provide evidence of the strong commitment of system leaders (clinical and non-clinical) to the strategy of HealthPathways implementation. Many clinical stakeholders were supportive of the implementation because they believed it helped decision-making and referrals. Leadership and well-developed pre-existing strong relationships across the health and social care economy were viewed as important for the sustainability of online care pathways and reflect findings from other sites outside the UK. ${ }^{26}{ }^{27}$ Improved communication through more face-toface contact in multidisciplinary education forums would support professional and organisational relationships and enhance engagement with HealthPathways. It would also be a clear pathway for referrals, with the potential to reduce tensions between primary and secondary care during a time of whole system change. ${ }^{28}$ Education and clinical engagement and collaboration has a prominent focus in NHS policy, ${ }^{29}$ with varying views on whether integrated care pathways are enablers to collaboration or vice versa. ${ }^{26} 3031$ Our work reflects the importance of clinical engagement and feedback in pathway development observed elsewhere. ${ }^{32} 33$

We used mixed methods to gain insight into the implementation of HealthPathways within this complex environment. The challenges of interpretation of context, mechanisms and outcomes configurations are discussed in the literature, particularly as realistic approaches are increasingly being used in health services implementation studies. ${ }^{23}{ }^{34-36}$ The decision, for example, of whether 'leadership' is a context, mechanism or outcome was explored with a purposive sample of HealthPathways users and non-users as well as clinical and non-clinical participants.

This study therefore focused not only on user perceptions of the implementation process but on the systems for development of HealthPathways and whether the pathways were being used. We found use of HealthPathways and clinicians' engagement with it was increasing. In particular, having local, complete pathways in a single repository motivated GPs to use the system. However, GPs and consultants who were acting as subject matter experts expressed concerns about the process of developing pathways in terms of consistency and speed. This meant that parts of HealthPathways were considered incomplete and therefore perceived to be inaccurate which may have influenced motivations to use and contribute to HealthPathways.

In HealthPathways sites outside the UK, additional facilitators and GP liaison officers work with GPs and clinicians $^{16}$ to support completion of pathways more rapidly. Analysis of the resources and process of pathway development identified that several services linked to specific pathways had not yet been configured. This meant that delivery was slower for some pathways, leading to additional time spent by clinical editors and subject matter experts, and potential loss of return on investment. Other studies have attempted to quantify the costs of clinician input into service development. ${ }^{37}$ The emerging role of clinicians' involvement as a potential transformer of services through the development of online care pathways requires further consideration in terms of the requirements and resources allocated. ${ }^{15}$

Users reported that HealthPathways was an important new resource for decision-making and referrals enabling them to carry out their role more effectively. Shared decision-making with patients is an important local and national policy driver, which can be supported by the use of online care pathways and resources. ${ }^{38}{ }^{39}$ Understanding the decision-making process through analysis of non-compliance or variance from agreed pathways was not within the scope of this study as data about referrals linked to agreed pathways were unavailable; however, several patient stories were cited with positive outcomes for patients. The influence of HealthPathways on shared decision-making requires further study.

Other researchers have found a reluctance to use guidelines and care pathways because there is often a perception that they are perceived to reduce professional judgement, personal focus on the patient and job satisfaction. ${ }^{640}$ Others have found endorsement for pathways, but subsequently little evidence of actual use. ${ }^{10}$ This was not the case in this study, where our early data indicated increasing use of HealthPathways. In future, users will have individual rather than site logins, enabling data to be gathered to correlate between users, referrals and 
outcomes. Other researchers have focused in more depth on the need for audit and analysis of variance in use of agreed pathways ${ }^{9} 378$; however, quantitative data of the kind collected in this study could provide an analysis of non-compliance with, or variance from, pathways and could also strengthen future studies and service development. ${ }^{264142}$ Ideally variance should be captured automatically as part of recording use.

\section{CONCLUSION}

This study has provided early findings in relation to the implementation of online care pathways across health and social care. The configurations provide a framework for considerations to be made for future implementations sites. We found most users engaged with the principle of online care pathways as a tool in their day-to-day work, seeing the benefit for their patients and their local system. System leaders were seeing sustained uptake and engagement, with access now also being provided for a wider group of health professionals including nurses.

As other sites in the UK begin to implement HealthPathways, there will be an opportunity for further research into online care pathways across the health and social care system, and to explore how context may affect implementation. New sites should ensure data collection and monitoring systems focus on outcomes (patient and care system) to establish return on investment and support future research in this area. Management of clinicians' expectations during the introductory phase could address tensions found between the ideal vision of HealthPathways voiced by system leaders and uncertainties voiced by users deriving from early practical experiences.

In New Zealand, a patient-accessible view of HealthPathways has been developed to support a partnership approach to care and treatment, with patients being able to view a pathway before they consult a relevant health professional or see their GP. In this study, initial patient stories recounted by clinicians provided useful insights into patient outcomes; however, further involvement of patients in research into care pathways may provide different perspectives and contribute to care pathway and service development.

Acknowledgements The authors thank the participants from general practices in South Tyneside, South Tyneside NHS Foundation Trust, South Tyneside Clinical Commissioning Group, North of England Commissioning Support in particular the support of Shona Haining and Mark Girvan, South Tyneside District Council, HealthNet (link group to voluntary care organisations), Streamliners NZ.

Contributors JA was principal investigator, carried out some of the interviews and final analysis and was responsible for leading the writing of the manuscript. ZS gathered interview and focus group data, provided initial analysis of themes using Nvivo and a draft of initial findings. IG carried out manual analysis of transcripts during final stages of the project and contributed to the writing of the manuscript. $\mathrm{JL}$ assisted with the analysis and contributed to the writing of the manuscript.

Funding Funding for this research was provided by North of England Commissioning Support (NECS) and Streamliners (supplier of HealthPathways) https://streamliners.co.nz/HealthPathways.aspx. NECS provided representatives for the research project steering group.
Disclaimer The views expressed in this article are those of the research team and not an official position of the institution or funder.

Competing interests $I G$ is married to a member of the executive team in South Tyneside Clinical Commissioning Group.

Patient consent for publication Not required.

Ethics approval Sunderland Research Ethics Committee and Health Research Authority (HRA).

Provenance and peer review Not commissioned; externally peer reviewed.

Data sharing statement Dataset available from the University of Sunderland respository SURE; access to data available on request.

Open access This is an open access article distributed in accordance with the Creative Commons Attribution Non Commercial (CC BY-NC 4.0) license, which permits others to distribute, remix, adapt, build upon this work non-commercially, and license their derivative works on different terms, provided the original work is properly cited, appropriate credit is given, any changes made indicated, and the use is non-commercial. See: http://creativecommons.org/licenses/by-nc/4.0/.

\section{REFERENCES}

1. NHS. NHS England five year forward view. https://www.england.nhs. uk/wp-content/uploads/2014/10/5yfv-web.pdf (accessed 14 Feb 2018).

2. NHS. NHS England workforce. https://www.england.nhs.uk/ integrated-care-pioneers/resources/workforce/ (accessed 14 Feb 2018).

3. Health Education England. Building a digital ready workforce. 2017 https://hee.nhs.uk/our-work/building-digital-ready-workforce (accessed 4 Jun 2018).

4. Blaser R, Schnabel M, Biber C, et al. Improving pathway compliance and clinician performance by using information technology. Int $\mathrm{J}$ Med Inform 2007;76:151-6.

5. Brennan N, Mattick K, Ellis T. The Map of Medicine: a review of evidence for its impact on healthcare. Health Info Libr J 2011;28:93-100.

6. Rooney E. Developing care pathways--lessons from the Steele Review implementation in England. Gerodontology 2014;31:52-9.

7. Rotter T, Kinsman L, James E, et al. Clinical pathways: effects on professional practice, patient outcomes, length of stay and hospital costs. Cochrane Database Syst Rev 2010;3:CD006632.

8. NHS. Next steps on the NHS five year forward view. https://www. england.nhs.uk/wp-content/uploads/2017/03/NEXT-STEPS-ONTHE-NHS-FIVE-YEAR-FORWARD-VIEW.pdf (accessed 14 Feb 2018)

9. Atwal A, Caldwell K. Do multidisciplinary integrated care pathways improve interprofessional collaboration? Scand J Caring Sci 2002;16:360-7.

10. CPA-Rapid-Review-Effectiveness-of-care-pathways.pdf. http://www cpa.org.uk/information/reviews/CPA-Rapid-Review-Effectiveness-ofcare-pathways.pdf (accessed 18 Feb 2018).

11. Hindle D, Yazbeck AM. Clinical pathways in 17 European Union countries: a purposive survey. Aust Health Rev 2005;29:94.

12. Horrocks S, Anderson E, Salisbury C. Systematic review of whether nurse practitioners working in primary care can provide equivalent care to doctors. BMJ 2002;324:819-23.

13. Grimshaw JM, Russell IT. Effect of clinical guidelines on medical practice: a systematic review of rigorous evaluations. The Lancet 1993;342:1317-22.

14. Charles A. Developing accountable care systems. https://www. kingsfund.org.uk/publications/developing-accountable-caresystems.

15. Timmins $\mathrm{N}$, Ham $\mathrm{C}$. The quest for integrated care in New Zealand. https://www.kingsfund.org.uk/sites/default/files/field/field publication_file/quest-integrated-care-new-zealand-timmins-hamsept13.pdf (accessed 14 Feb 2018).

16. HealthPathways Community. HP Community. https://www.healthpa thwayscommunity.org/ (accessed 14 Feb 2018).

17. Alison Boughey Consulting HealthPathways: An evaluation of its implementation in five Australian Medicare Locals. http://www.alis onbougheyconsulting.com.au/wordpress/wp-content/uploads/2013/ 01/Alison-Boughey-Consulting-AML-Alliance-HealthPathwaysevaluation-final-report.pdf (accessed 14 Feb 2018).

18. McGeoch G, McGeoch P, Shand B. Is HealthPathways effective? An online survey of hospital clinicians, general practitioners and practice nurses. N Z Med J 2015;128:1408.

19. England NHS. Integrated care pioneers. https://www.england.nhs.uk/ integrated-care-pioneers/ (accessed 14 Feb 2018). 
20. Pawson R, Tilley N. Realistic Evaluation. London: Sage.

21. Pawson R, Greenhalgh T, Harvey G, et al. Realist review--a new method of systematic review designed for complex policy interventions. J Health Serv Res Policy 2005;10:21-34.

22. Moore GF, Audrey S, Barker M, et al. Process evaluation of complex interventions: medical research council guidance. BMJ 2015;350:h1258.

23. Rycroft-Malone J, Fontenla M, Bick D, et al. A realistic evaluation: the case of protocol-based care. Implement Sci 2010;5:38.

24. Ogrinc G, Davies L, Goodman D, et al. SQUIRE 2.0 (Standards for QUality Improvement Reporting Excellence): revised publication guidelines from a detailed consensus process. Am J Med Qual 2015;30:543-9.

25. Røsstad T, Garåsen $H$, Steinsbekk A, et al. Implementing a care pathway for elderly patients, a comparative qualitative process evaluation in primary care. BMC Health Serv Res 2015;15:86.

26. Deneckere S, Euwema M, Van Herck P, et al. Care pathways lead to better teamwork: results of a systematic review. Soc Sci Med 2012;75:264-8. 1.

27. Greenhalgh T, Robert G, Macfarlane F, et al. Diffusion of innovations in service organizations: systematic review and recommendations. Milbank Q 2004;82:581-629.

28. Brown BB, Patel C, Mclnnes E, et al. The effectiveness of clinical networks in improving quality of care and patient outcomes: a systematic review of quantitative and qualitative studies. BMC Health Serv Res 2016;16:360.

29. NHS. Developing people improving care. https://improvement.nhs. uk/documents/542/Developing_People-Improving_Care-010216.pdf (accessed 6 Jun 2018).

30. West M, Eckert R, Steward K, et al. Developing collective leadership for health care. 36.

31. Evans-Lacko S, Jarrett M, McCrone P, et al. Facilitators and barriers to implementing clinical care pathways. BMC Health Serv Res 2010;10:182.
32. Robinson S, Varhol R, Bell C, et al. HealthPathways: creating a pathway for health systems reform. Aust Health Rev 2015;39:9-11.

33. Mansfield SJ, Quirk F, von Treuer K, et al. On the right path? Exploring the experiences and opinions of clinicians involved in developing and implementing HealthPathways Barwon. Aust Health Rev 2016;40:129-35

34. Greenhalgh T, Wong G, Jagosh J, et al. Protocol--the RAMESES II study: developing guidance and reporting standards for realist evaluation. BMJ Open 2015;5:e008567.

35. Gagliardi AR, Marshall C, Huckson S, et al. Developing a checklist for guideline implementation planning: review and synthesis of guideline development and implementation advice. Implement Sci 2015;10:19.

36. Lacouture A, Breton E, Guichard A, et al. The concept of mechanism from a realist approach: a scoping review to facilitate its operationalization in public health program evaluation. Implement Sci 2015;10:153.

37. McLaughlin N, Burke MA, Setlur NP, et al. Time-driven activity-based costing: a driver for provider engagement in costing activities and redesign initiatives. Neurosurg Focus 2014;37:E3.

38. NHS. RightCare shared decision making programme. https://www. england.nhs.uk/rightcare/shared-decision-making/ (accessed 4 Jun 2018).

39. Coulter A, Edwards A, Elwyn G, et al. Implementing shared decision making in the UK. Z Evid Fortbild Qual Gesundhwes 2011;105:300-4.

40. Woolf SH, Grol R, Hutchinson A, et al. limitations, and harms of clinical guidelines. BMJ 1999;318:527-30.

41. Croucher M. An evaluation of the quality of integrated care pathway development in the UK national health service. Journal of integrated Care Pathways 2005;9:6-12.

42. Campbell H, Hotchkiss R, Bradshaw N, et al. Integrated care pathways. BMJ 1998;316:133-7. 